(1)

CrossMark

\title{
Pandemic trials: evidence-based medicine on steroids
}

\author{
James D. Chalmers \\ Affiliation: Division of Molecular and Clinical Medicine, Ninewells Hospital, Dundee, UK.
}

Correspondence: James D. Chalmers, Division of Molecular and Clinical Medicine, Ninewells Hospital, Dundee, DD1 9SY, UK. E-mail: jchalmers@dundee.ac.uk

@ERSpublications

Small trials have been largely overlooked in the early part of the COVID-19 pandemic in favour of "mega-trials". As new therapies are urgently needed, phase 2 "proof of concept" studies are crucial as we move into a new phase of pandemic research. https://bit.ly/3o0K9kl

Cite this article as: Chalmers JD. Pandemic trials: evidence-based medicine on steroids. Eur Respir J 2020; 56: 2004116 [https://doi.org/10.1183/13993003.04116-2020].

Research is the only exit strategy from current coronavirus disease 2019 (COVID-19) restrictions. The second wave of COVID-19 infections currently affecting most of Europe emphasises that COVID-19, the disease caused by infection with severe acute respiratory syndrome coronavirus 2 (SARS-CoV-2), is likely to require strict control measures impacting on the daily life of most citizens until either a highly effective approach to treatment or effective vaccines are in widespread use [1-3].

Management approaches include prophylaxis, treatments that aim to prevent infection, for example in high risk groups such as healthcare workers [4] or those recently exposed to infected individuals [5], and treatment of established disease [6]. Among the treatment approaches for established disease, these can be broadly divided into those that target the virus either, for example, through direct antiviral activity or interfering with virus access to host cells, and those that target the host immune response. Evidence is accumulating that much of the morbidity and mortality associated with COVID-19 disease is related to a dysregulated host immune response and it has been described and clinically observed that COVID-19 often follows a biphasic pattern with an initial viral phase followed in susceptible individuals with a second "host inflammatory" phase that may be associated with respiratory failure and the development of acute respiratory distress syndrome [7-9].

It is urgent that effective therapies are found, and a dizzying number of trials have been initiated since the start of the pandemic. Indeed, the World Health Organization (WHO) database lists more than 2500 trials worldwide at the last count. Many of these trials are small and several overlap in terms of their interventions and outcomes. For example, more than 150 unique trials of hydroxychloroquine were initiated during the early part of the pandemic [10].

The most notable feature of the pandemic has been the impact of the pragmatic "mega-trials" like RECOVERY and SOLIDARITY [11-13]. These large, open-label trials which are embedded within routine clinical practice test a number of different interventions simultaneously using streamlined platform designs incorporating a "standard care" comparator without blinding. Broad inclusion criteria allow large numbers of patients to be enrolled rapidly and as of 8 November, 2020 RECOVERY had enrolled more than 16000 patients while SOLIDARITY, sponsored by the WHO, recently reported data from 11266 patients in their initial report of repurposed antiviral drugs [11, 13-15]. 
RECOVERY has demonstrated that both hydroxychloroquine and lopinavir-ritonavir are ineffective, and results of azithromycin, tocilizumab and convalescent plasma arms are expected shortly [11, 14, 15]. SOLIDARITY found no efficacy in terms of the primary outcome of mortality for any of the interventions tested, namely hydroxychloroquine (rate ratio (RR) 1.19, 95\% CI 0.89-1.59), lopinavir-ritonavir (RR 1.00, 95\% CI $0.79-1.25$ ), interferon- $\beta$ (RR 1.16, 95\% CI 0.96-1.39) or remdesivir (RR 0.95, 95\% CI 0.81-1.11) [13]. The sample sizes for hydroxychloroquine (947 versus 906) and lopinavir-ritonavir (1399 versus 1372) were lower than originally planned as these arms were terminated after RECOVERY found these drugs to be ineffective.

Dexamethasone, which was shown to reduce mortality in hospitalised patients in RECOVERY (RR 0.83, 95\% CI 0.75-0.93), particularly in patients requiring mechanical ventilation (RR 0.64, 95\%CI 0.51-0.81), remains at the time of writing the only intervention proven to reduce mortality in COVID-19 [11].

Whether this mortality benefit extends to other steroids has recently been investigated in a prospective meta-analysis of randomised trials by the WHO REACT working group [16]. They identified trials of dexamethasone, hydrocortisone and methylprednisolone and reported a reduced mortality for critically ill patients treated with corticosteroids ( OR 0.70, 95\% CI 0.48-1.01; p=0.053), albeit nominally not statistically significant and heavily influenced by the RECOVERY trial [16]. There was little inconsistency, suggesting an effect that is shared between different corticosteroids.

In the era of "mega-trials" what is the role of smaller proof of concept trials? In this issue of the European Respiratory Journal, EDALATIFARD et al. [17] report the results of a small, single-blind randomised trial of intravenous methylprednisolone in severe COVID-19. Patients in the study were randomised 1:1 to 3 days of $250 \mathrm{mg} \cdot \mathrm{day}^{-1}$ pulsed methylprednisolone or standard care. 34 patients were randomised in each group. The authors aimed to include patients with evidence of respiratory failure by including patients with oxygen saturations $<90 \%$ on air, elevated C-reactive protein and interleukin- 6 in the blood, and confirmed SARS-CoV-2 infection [17].

While a randomised study, the trial had significant limitations including a primary outcome of discharge or death. This combination of positive and negative outcomes in a single measure is unusual at best and probably even inappropriate. The authors excluded six patients from the "overall analysis" of the standard care arm because they received steroids, when usual practice would be to include these patients in the intention to treat analysis and exclude from a per protocol analysis. Nevertheless, the results of the meaningful secondary endpoints are striking. Time to clinical improvement was approximately 4.5 days shorter with methylprednisolone, and mortality was strikingly reduced (5.9\% versus $42.9 \%)$. The frequency of serious adverse events was similar between the groups.

The effect estimate in the study, particularly with regard to mortality, is greater than in any of the studies included in the recent meta-analysis, but with a high degree of uncertainty given the small sample size. The trial nevertheless supports the conclusions of the REACT meta-analysis that the mortality benefits of corticosteroids likely extends to these drugs as a class, rather than being limited to dexamethasone [16]. The trial also provides welcome data on the safety of steroids, which were not reported in RECOVERY. Despite these positive features, the design limitations, a small sample size, the use of hydroxychloroquine, lopinavir and naproxen as routine background therapy and other design issues mean this study should be treated with caution.

In the era of "mega-trials" it is important to ask ourselves the question of whether small trials like this have a role. Should all efforts be focused on recruitment to large scale platforms? My answer would currently be no. In particular, the negative results from SOLIDARITY illustrated that drugs selected largely on the basis of theoretical considerations are likely to have a low success rate in large scale studies. The investment of time, cost and also patient exposure in testing a drug in thousands of patients requires more than just good in vitro antiviral activity or sound theory. Phase 2 studies to demonstrate preliminary evidence of efficacy and safety will become more and more important as the pandemic progresses and the experience with corticosteroids provides a useful argument in favour of this [18]. Most of the smaller studies conducted to date would point towards a beneficial effect of steroids: in other words, if dexamethasone or methylprednisolone were a previously unknown agent, testing in a small phase 2 study would have given sufficient evidence of safety and efficacy to provide confidence regarding incorporating steroids into "mega-trials" [16]. This provides confidence for doing this more going forward. Studies with sample sizes ranging from 60 to 300 may provide sufficient proof of concept, and crucially safety, to prioritise which agents then move into larger scale testing using platforms. Phase 2 platform trials with standardised protocols and central co-ordination, such as the ACCORD programme in the UK, provide a means to evaluate early-phase drugs in an efficient way [18].

The counter argument is that bigger is always better and that large trials may identify a signal that small trials could miss. In this regard, tocilizumab, an interleukin-6 receptor antagonist, will shortly be a 
powerful example. Three randomised trials have recently been published showing no benefit of tocilizumab on clinical outcomes in hospitalised patients. STONE et al. [19] enrolled 243 patients and found no significant effect on the primary outcome of intubation or death (hazard ratio $0.83,95 \%$ CI $0.38-1.81$; $\mathrm{p}=0.64$ ), Salvarani et al. [20] enrolled 126 patients and showed no effect on clinical worsening (RR 1.05, 95\% CI 0.59-1.86), while Hermine et al. [21] enrolled 131 patients and found no significant effect on any endpoint. Each had a modest sample size, but none showed convincing efficacy on primary or secondary endpoints and there are additional unpublished trials also showing lack of efficacy of this intervention. Despite this, RECOVERY continues to enrol patients into its comparison of tocilizumab versus standard care. Will the inclusion of 1500-2000 patients allow RECOVERY to find a signal that the small trials miss? From an evidence-based medicine and strategy perspective moving into the next phase of the pandemic, this may prove to be a crucial examplar.

Today the message is clear. There have never been more randomised trials globally, and the 2020 SARS-CoV-2 pandemic may come to be regarded as evidence-based medicine's finest hour if it can deliver the treatments we need. Corticosteroids are currently the only therapy proven to reduce mortality in patients hospitalised with COVID-19. Repurposed antiviral treatments have so far proven ineffective in reducing mortality. New therapies will need to be identified, prioritised and moved into large scale platform trials in the coming months. We have evidence for steroids, but to generate treatment protocols that will make a powerful impact on the pandemic we need more, which means enhancing phase 2 trial capability and feeding the best candidates into large scale platforms. We need evidence based medicine, on steroids.

Conflict of interest: J.D. Chalmers reports grants and personal fees from AstraZeneca, Boehringer Ingelheim, GlaxoSmithKline and Insmed, personal fees from Chiesi, Novartis and Zambon, grants from Gilead Sciences, outside the submitted work.

Support statement: This work is supported by the British Lung Foundation (GSK/BLF Chair of Respiratory Research). Funding information for this article has been deposited with the Crossref Funder Registry.

\section{References}

1 Lonergan $\mathrm{M}$, Chalmers J. Estimates of the ongoing need for social distancing and control measures post-"lockdown" from trajectories of COVID-19 cases and mortality. Eur Respir J 2020; 56: 2001483.

2 Guan W-J, Chen R-C, Zhong N-S. Strategies for the prevention and management of coronavirus disease 2019. Eur Respir J 2020; 55: 2000597.

3 Guan W-J, Ni Z-Y, Hu Y, et al. Clinical characteristics of coronavirus disease 2019 in China. N Engl J Med 2020; 382: $1708-1720$.

4 Siddiqui MK, Parcell B, Allstaff S, et al. Characteristics and outcomes of health and social care workers testing positive for SARS-CoV-2 in the Tayside region of Scotland. Eur Respir J 2020; 56: 2002568.

5 Lou B, Li T-D, Zheng S-F, et al. Serology characteristics of SARS-CoV-2 infection after exposure and post-symptom onset. Eur Respir J 2020; 56: 2000763.

6 Matthay MA, Aldrich JM, Gotts JE. Treatment for severe acute respiratory distress syndrome from COVID-19. Lancet Respir Med 2020; 8: 433-434.

7 McElvaney OJ, McEvoy N, McElvaney OF, et al. Characterization of the inflammatory response to severe COVID-19 illness. Am J Respir Crit Care Med 2020; 202: 812-821.

8 Remy KE, Mazer M, Striker DA, et al. Severe immunosuppression and not a cytokine storm characterize COVID-19 infections. JCI Insight 2020; 5: e140329.

9 Chalmers JD, Chotirmall SH. Rewiring the immune response in COVID-19. Am J Respir Crit Care Med 2020; 202: 784-786.

10 Mathioudakis AG, Fally M, Hashad R, et al. COVID-19 clinical trials: unravelling a methodological Gordian knot. Am J Respir Crit Care Med 2020; 202: 635-637.

11 Horby P, Lim WS, Emberson JR, et al. Dexamethasone in hospitalized patients with Covid-19 - preliminary report. N Engl J Med 2020; in press [https://doi.org/10.1056/NEJMoa2021436].

12 Bai C, Chotirmall SH, Rello J, et al. Updated guidance on the management of COVID-19: from an American Thoracic Society/European Respiratory Society coordinated international task force. Eur Respir Rev 2020; 29: 200287.

13 WHO Solidarity trial consortium, Pan H, Peto R, et al. Repurposed antiviral drugs for COVID-19 - interim WHO SOLIDARITY trial results. medRxiv 2020; preprint [https://doi.org/10.1101/2020.10.15.20209817].

14 RECOVERY Collaborative Group. Lopinavir-ritonavir in patients admitted to hospital with COVID-19 (RECOVERY): a randomised, controlled, open-label, platform trial. Lancet 2020; 396: 1345-1352.

15 Horby P, Mafham M, Linsell L, et al. Effect of hydroxychloroquine in hospitalized patients with Covid-19. N Engl J Med 2020; 383: 2030-2040.

16 Sterne JAC, Murthy S, Diaz JV, et al. Association between administration of systemic corticosteroids and mortality among critically ill patients with COVID-19: a meta-analysis. JAMA 2020; 324: 1330-1341.

17 Edalatifard M, Akhtari M, Salehi M, et al. Intravenous methylprednisolone pulse as a treatment for hospitalised severe COVID-19 patients: results from a randomised controlled clinical trial. Eur Respir J 2020; 56: 2002808.

18 Wilkinson T, Dixon R, Page C, et al. ACCORD: a multicentre, seamless, phase 2 adaptive randomisation platform study to assess the efficacy and safety of multiple candidate agents for the treatment of COVID-19 in hospitalised patients: a structured summary of a study protocol for a randomised controlled trial. Trials 2020; 21: 691 . 
19 Stone JH, Frigault MJ, Serling-Boyd NJ, et al. Efficacy of tocilizumab in patients hospitalized with Covid-19. N Engl J Med 2020; in press [https://doi.org/10.1056/NEJMoa2028836].

20 Salvarani C, Dolci G, Massari M, et al. Effect of tocilizumab vs standard care on clinical worsening in patients hospitalized with COVID-19 pneumonia: a randomized clinical trial. JAMA Intern Med 2020; in press [https://doi. org/10.1001/jamainternmed.2020.6615].

21 Hermine O, Mariette X, Tharaux P-L, et al. Effect of tocilizumab vs usual care in adults hospitalized with COVID-19 and moderate or severe pneumonia: a randomized clinical trial. JAMA Intern Med 2020; in press [https://doi.org/10.1001/jamainternmed.2020.6820]. 